\title{
El conocimiento apriorístico y la enseñanza de la economía
}

\section{A Priori Knowledge and Teaching of Economics}

\author{
Jorge Iván González*
}

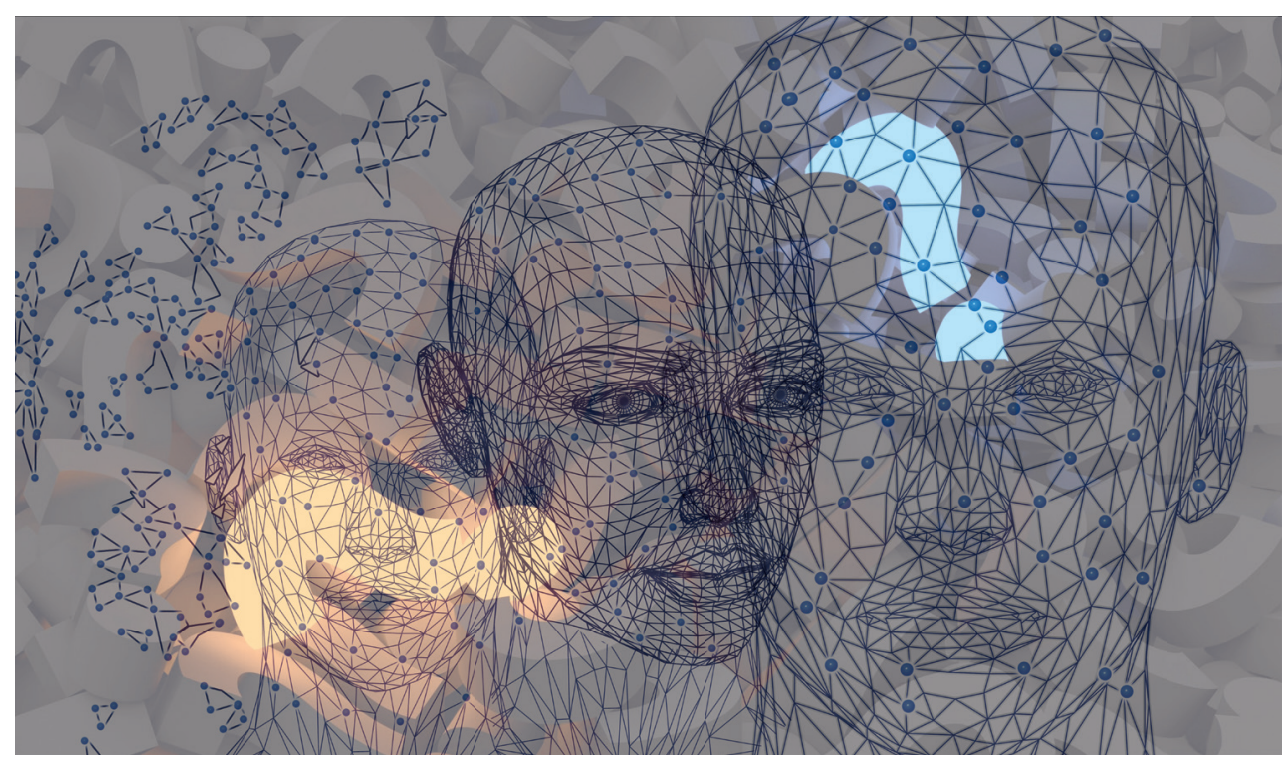

\section{Resumen}

El artículo comienza con un texto de Keynes sobre la naturaleza del pensamiento económico, en el que menciona tres fases del proceso cognitivo: i) el pensamiento ordenado, ii) la desagregación de las causalidades parciales, iii) la mirada multicausal del fenómeno. A partir de allí, se explica que la pretensión de neutralidad valorativa sigue alimentando la vana ilusión del conocimiento objetivo. A pesar de que en la historia del pensamiento la crítica al positivismo ha sido intensa, la enseñanza de la economía se niega a reconocer la presencia inevitable de los prejuicios. Y al negar que todo conocimiento está fundado en nociones apriorísticas, termina absolutizando el método y ahogando el pensamiento.

Palabras clave: conocimiento apriorístico, enseñanza de la economía, crítica al positivismo, absolutización del método.

Citar este artículo como: González, J. I. (2020). El conocimiento apriorístico y la enseñanza de la economía. Revista Papeles, 12(24), 15-26.

Fecha de recibido: enero 15 de 2020. Fecha de aceptado: marzo 25 de 2020.

* Profesor de las universidades Nacional y Externado. Ph. D. en economía de la Universidad Católica de Lovaina. MA en economía de la Universidad de los Andes. Filósofo de la Universidad Javeriana, Correo electrónico: jorgeivangonzalez29@gmail.com 


\begin{abstract}
This paper begins with Keynes' text on the nature of economic thought where he states three stages of the cognitive process: i) ordered thought; ii) the disintegration of partial causalities, iii) the multi-causal perspective of phenomena. From there on, I explain that the value neutrality claim still gives the vain illusion of objective knowledge. Although there has been severe criticism of positivism in the history of economic thought, schools and economic professors still refuse to acknowledge the inevitable existence of prejudices. To deny that all knowledge is based on a priori notions absolutizes the method and stops thinking.
\end{abstract}

Keywords: a priori knowledge, economics teaching, criticism of positivism, absolutism of the method.

\title{
1. Introducción
}

Es frecuente que se invoque la neutralidad valorativa como principio rector de la enseñanza y de la investigación en economía. Este camino está llevando a callejones sin salida. La negación de los prejuicios es inútil porque siempre están presentes. La enseñanza de la economía tiene que reconocer la existencia de juicios de valor, y debe crear las condiciones apropiadas para que las preguntas que mueven el conocimiento sean relevantes.

El artículo comienza con una reflexión keynesiana sobre la naturaleza del pensamiento económico. $\mathrm{Y}$ en las secciones siguientes desarrolla cada uno de sus componentes: el a priori, la desagregación causal y la integración multicausal. Al final se hace una breve reflexión sobre la verdad y el conocimiento.

El texto está marcado por un profundo escepticismo sobre la enseñanza de la economía, y sobre el método de conocer. En lugar de superar los problemas que se han identificado hace años, los males parecen agudizarse, $y$ tanto docentes como estudiantes padecen las angustias derivadas de la absolutización del método, y del abandono del pensamiento crítico y diversificado.

\section{La naturaleza del pensamiento económico}

La enseñanza de la disciplina debe estar centrada en profundizar en "la naturaleza del pensamiento económico”, que Keynes define así:

El propósito de nuestro análisis no es proporcionar una máquina, o un método de manipulación ciega que permita obtener una respuesta infalible. El objetivo es ofrecer un método de pensamiento ordenado que contribuya a pensar nuestros problemas particulares. Y después de que hayamos llegado a una conclusión provisional, gracias a que hemos aislado, uno a uno, los factores complejos, podremos regresar sobre nosotros mismos y tratar de encontrar, hasta donde sea posible, la interacción entre estos factores. Esta es la naturaleza del pensamiento económico (Keynes 1936, pos. 5227).

Los elementos constitutivos de esta definición son: i) pensamiento ordenado, ii) análisis desagregado e iii) interacción multifactorial. Y advierte Keynes que este tipo de aproximación no tiene nada que ver con interpretaciones mecánicas, o con la manipulación de instrumentos ciegos que pretenden llegar a respuestas "infalibles". 
El método de pensamiento ordenado es el punto de partida de todo proceso cognitivo. Es el a priori subjetivo que permite jerarquizar los hechos sociales, y formular las hipótesis iniciales. La aceptación de este postulado es, por sí misma, una crítica al positivismo. La persona no se acerca a la realidad de manera neutra esperando que esta envíe sus mensajes de forma objetiva. No es posible conocer sin un pre-ordenamiento subjetivo. El sujeto, con su historia y sus prejuicios, juzga qué se debe investigar. Y, aún más, se aproxima a los hechos con unos instrumentos metodológicos prediseñados. Los juicios de valor permean la selección del problema y la forma de abordarlo.

La escogencia del problema relevante es un insumo central del análisis económico. Con el paso del tiempo se han ido consolidando las herramientas analíticas que permitieron abordar un asunto que en su momento se juzgó importante. El avance de la economía, dice Schumpeter (1978), está directamente relacionado con el fortalecimiento de los instrumentos analíticos. Los principios constitutivos de la disciplina correspondieron a problemas que en su momento se juzgaron relevantes. Y la gran búsqueda de los economistas, a la que se refiere Nasar (2011), está animada por el propósito de entender los hechos sociales para transformarlos. Y este afán de corregir males, como los que se vivían en el siglo XIX, fue una preocupación

\section{El conocimiento apriorístico}

La enseñanza de la economía debería llevar al estudiante a preguntarse por los problemas que son relevantes. Abundan las reflexiones, las estimaciones y los datos sobre asuntos que no son importantes. Y como la pertinencia de un tema depende de numerosas circunstancias, no existe ninguna fórmula para acertar. En lugar de pretender un conocimiento "objetivo", "libre de valores", la formación del economista tiene que llevar a reconocer los prejuicios de de Marx, Mill, George, Edgeworth, Marshall, Walras...

Una vez que se ha definido el problema, el siguiente paso es llegar a una conclusión provisional gracias al ejercicio de diferenciación. Y en este proceso se construyen relaciones de causalidad que siempre son parciales. En las disciplinas sociales ninguna relación causaefecto es unidireccional. Pero es necesario hacer taxonomías e imaginar secuencias causales. Y este ejercicio ofrece las primeras pistas para la interpretación del fenómeno que se ha juzgado importante. Cualquier hipótesis es provisional. Su transitoriedad se explica porque la aproximación es fragmentada y, por tanto, imperfecta y limitada.

El tercer momento consiste en mostrar la interacción entre factores. Es la recuperación del análisis multicausal. La argumentación de Keynes en La Teoría General muestra la complejidad de la interrelación de las distintas variables. Nada es unicausal. Por ejemplo, en su función de demanda presenta determinantes "objetivos" y "subjetivos". En su análisis de la tasa de interés explicita la interacción entre los precios esperados y los actuales. La tasa de interés de cada bien (trigo, casa, máquinas...), es el resultado de múltiples factores. $\mathrm{Y}$ en sus reflexiones vuelve, una y otra vez, sobre la relación entre el salario y la propensión marginal a consumir. Ninguna de las causalidades propuestas es la Teoría General es unidireccional. cada uno, y a evaluarlos. Aceptando que la dimensión valorativa siempre está presente, la tarea es ordenar el pensamiento tratando de indagar por aquello que se considera relevante. $\mathrm{Y}$ en este proceso, lo más obvio es preguntarse por la razón de la escogencia del tema, y por las implicaciones que se podrían derivar de su conocimiento. Esta afirmación es válida, ante todo, para el docente, que selecciona el curso, la bibliografía, y los énfasis. El 
La reflexión sobre la enseñanza tiene que preguntarse por los a priori que permiten avanzar en el conocimiento. Sin estos prejuicios no es posible construir hipótesis, y el método termina ahogando la reflexión. profesor enseña lo que sabe. Y esta escogencia es el resultado de sus a priori. De sus juicios de valor. Así que cuando en el mundo académico los profesores se interesan por asuntos que no son relevantes, la enseñanza no contribuye a que los estudiantes se hagan las preguntas pertinentes.

Ya no tiene mucho sentido, por ejemplo, continuar anclados en los rendimientos constantes de una función de producción Cobb y Douglas (1928). Es inaceptable que después de casi 100 años, los modelos de crecimiento y de producción sigan enfrascados en la lógica de la Cobb-Douglas, e insistan en el supuesto de los rendimientos decrecientes del factor. Tampoco parece relevante volver sobre la concavidad de la función de utilidad de Samuelson (1937). El supuesto de no saciabilidad, ya debería ser asunto del pasado ${ }^{1}$.

En lugar de estos temas, que siguen copando gran parte de la enseñanza, se debe volver a preguntas básicas, como la distribución, que preocupó tanto a los pensadores del siglo XIX²,

1 De la misma manera, no tiene sentido continuar afirmando que la demanda depende exclusivamente del ingreso. Por el lado del enfoque marxista, han dejado de ser importantes las reflexiones sobre la diferencia entre trabajo productivo/improductivo, o sobre la transformación de valores a precios. La historia del debate de la transformación de valores a precios, la describe muy bien Cuevas (1986). La pregunta por el valor de las mercancías ha sido relevante desde Aristóteles y continuará siéndolo.

2 Edgeworth (1879, p. 394) expresa bien el espíritu distributivo del siglo XIX. El principal problema y que ahora vuelve a ser relevante ${ }^{3}$. También han ido ganando importancia los aspectos relacionados con la geografía (Krugman 1991, Duranton y Puga 2004), con la sicología (Kahneman 2002, 2011; Thaler 2017) y la biología (Simon $1978 a, b$ ).

Estas enumeraciones apenas son ilustrativas. Cada comunidad académica debe ir moldeando los prejuicios de sus miembros, buscando que en el debate se vayan creando las condiciones para que el método de pensamiento sea ordenado. El diálogo entre pares puede evitar que el docente se pierda en una maraña de asuntos irrelevantes.

La reflexión sobre la enseñanza tiene que preguntarse por los a priori que permiten avanzar en el conocimiento. Sin estos prejuicios no es posible construir hipótesis, y el método termina ahogando la reflexión. La abundancia de datos puede llevar a afirmaciones tan vacías, por no decir estúpidas, como “... las personas de nombre José, y que tienen entre 20 y 30 años, son las que más probabilidad tienen de no pagar el crédito". $\mathrm{Y}$, precisamente, una de las dificultades de la big data, y de la sofisticación de los métodos, es la destrucción del pensamiento y de la reflexión crítica, que únicamente son posibles cuando los a priori llevan a la formulación de preguntas pertinentes.

Todavía hay quienes argumentan que se debe buscar la "neutralidad" valorativa. Y que la técnica permite eliminar los sesgos de los investigadores. Esta pretensión positivista le ha hecho mucho daño a la disciplina, y ha castrado la discusión. Es notorio el empobrecimiento del debate entre economistas. Incluso, al interior de las distintas universidades. Cada quien toma las decisiones sobre lo que

del Cálculo Hedónico es “...encontrar (i) la distribución de medios y (ii) de trabajo, la (iii) calidad y el (iv) número de personas, de tal manera que se logre la mayor felicidad posible".

3 De diversas maneras, Piketty $(2013,2019)$ insiste en la actualidad del tema distributivo. 
considera pertinente, y sin que medie la más mínima discusión académica, lo transmite a los estudiantes. Se va generando, entonces, una cadena de especialistas en metodología que no reflexionan, y que terminan agobiados en sus esfuerzos por perfeccionar la técnica. $Y$ el proceso tiene su punto final en las revistas de prestigio que también están rindiéndole un excesivo culto al método, dejando de lado el razonamiento.

Marshall, que tuvo mucha influencia en Keynes, es claro sobre la importancia del sentido común, en la construcción del proceso cognitivo.

Hemos visto que el economista tiene que examinar los hechos, pero los hechos por sí mismos no dicen nada. La historia nos ofrece secuencias y coincidencia, pero solamente la razón puede interpretarlos y sacar lecciones de ellos. Para lograr este propósito se debe hacer un trabajo arduo, en el que el sentido común se convierte en el último árbitro para resolver cada problema práctico. La ciencia económica no es sino el resultado del sentido común ayudado por un análisis organizado $y$ por el ejercicio de la razón, que facilita la tarea de recoger, jerarquizar, y esbozar inferencias a partir de los hechos particulares. Aunque los alcances del sentido común sean limitados, sin su ayuda el trabajo cognitivo sería en vano (Marshall, 1994, p. 32).

El mensaje es contundente. En lugar de negar el a priori, la enseñanza de la economía lo tiene que cultivar, de tal manera que, en el proceso de interacción de cada comunidad académica, las preguntas de investigación sean relevantes. $\mathrm{Y}$ en este ejercicio de desarrollar el sentido común es fundamental el diálogo interpares. La enseñanza de la disciplina debería ofrecer los elementos que permitan detectar los problemas básicos.

Aunque la definición de las preguntas de investigación que sean pertinentes es un reto enorme de cualquier comunidad académica, un primer paso, relativamente sencillo, consiste en revisar con nuevos ojos los artículos y las tesis de maestría y doctorado, para concluir rápidamente que en la mayoría de estos trabajos la predominancia del método ha aniquilado el pensamiento, y que el sentido común ha desaparecido en el proceso árido de perfeccionar los procedimientos formales. El método por el método corroe los espíritus, y lleva a que estudiantes y profesores pierdan el interés, en el lenguaje de Nasar, por la gran búsqueda.

Un estudiante juicioso me dice que hará su tesis para indagar cuál es la probabilidad de que una persona del sector informal no se pensione. $Y$ para responder esta inquietud presenta 4 sofisticados modelos alternativos. Al preguntarle cuál se imagina que sería el resultado esperado, me responde que, efectivamente, las personas del sector informal tienen poca probabilidad de pensionarse, pero que quiere comparar los métodos. Le digo que quizá con el método $\mathrm{A}$ le dé $80 \%$, con el B $75 \%$, con el C $83 \%$, y con el D $90 \%$. Cualquiera que sea el resultado,

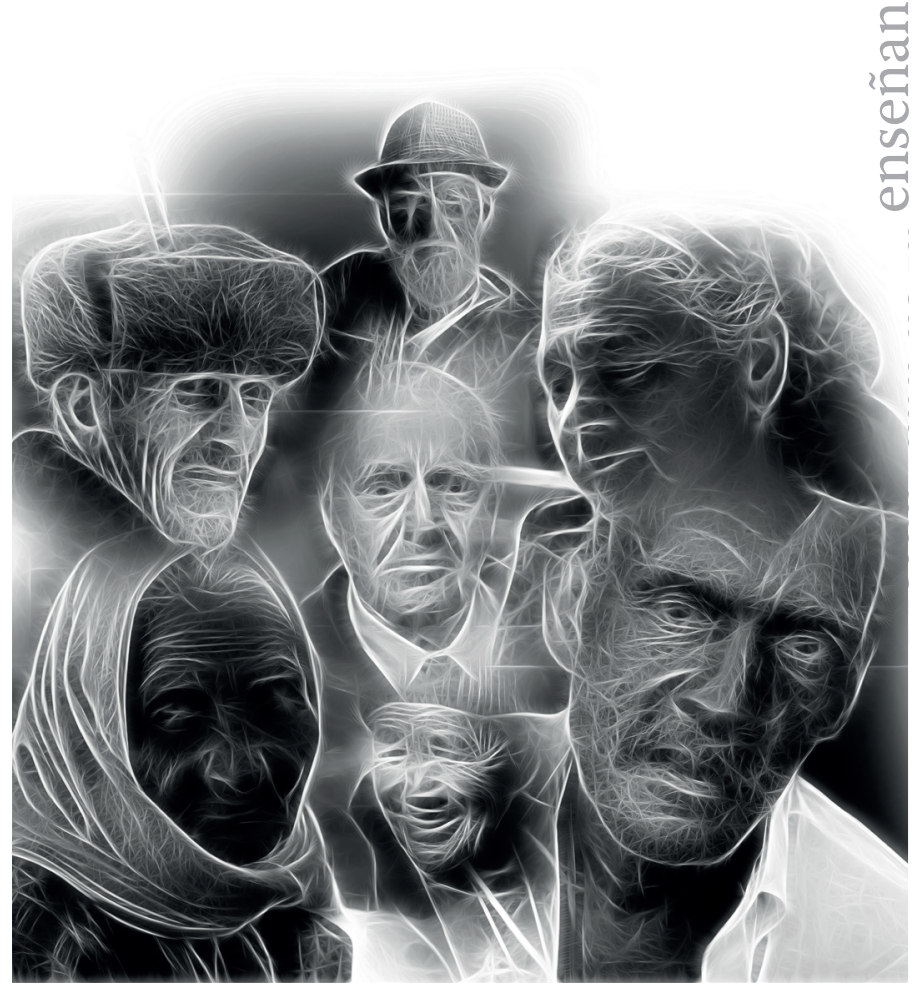


ninguno es relevante. La pregunta importante, que no se hace el estudiante y tampoco su director, debería ser de este estilo ¿cómo lograr que las personas del sector informal puedan tener una vejez decente? Esta reflexión que sí es interesante, y que es intrínsecamente compleja, requiere un conocimiento apriorístico que el estudiante, y el director, no tienen. La formación en la universidad no le ha permitido - ni al estudiante, ni a su director - construir prejuicios que le lleven a percibir en la realidad, como diría Marshall, aquellos hechos que ameritan un estudio sistemático.

La anécdota no es un caso único. En la disciplina abundan los estudios inútiles y la cuantificación elegante de relaciones obvias. Y la proliferación de estos trabajos, completamente alejados del sentido común, se explica por las siguientes razones: i) la ilusión positivista; ii) la preponderancia de universidades y revistas de élite; iii) la ausencia del debate al interior de la disciplina; iv) la compatibilidad entre las instancias de poder y la adoración del método.

La ilusión positivista lleva a pensar que el método es neutro y que permite captar la realidad externa con objetividad. Se supone que el hecho investigado se puede desprender de los elementos subjetivos del investigador ${ }^{4}$. En el lenguaje de profesores y estudiantes es frecuente escuchar afirmaciones del tipo: “... este procedimiento evita el sesgo político”, “... la realidad es así”, "... la causalidad de Granger es robusta”, “... actúo como técnico y no como político”, “... el resultado es contundente porque la muestra es representativa", “... aunque el hallazgo sea contra intuitivo, el coeficiente indica que esta relación es válida".

Y la ilusión positivista lleva a la falsa creencia que la ausencia de juicios de valor permite un acercamiento privilegiado a la verdad. Este vínculo, entre la negación de los prejuicios y la verdad, no es cierto. La verdad es una construcción colectiva que está mediada por el contraste de múltiples hipótesis y ejercicios empíricos. La realidad y la verdad no están por fuera de los sujetos. Las verdades, que siempre son parciales, resultan de la discusión colectiva de individuos con prejuicios, y no de la ausencia de juicios de valor.

Dicho de otra manera, no hay que temerle al a priori. No hay que rechazar los juicios de valor, sino que hay que recrearlos permanentemente en el diálogo académico interpares.

\section{La desagregación}

De la frase de Keynes se desprende un segundo momento. Una vez que los datos han sido organizados gracias al conocimiento apriorístico, se inicia el proceso de reflexión sobre las causalidades parciales. El proceso cognitivo obliga a buscar la relación causa-efecto de los fenómenos escogidos. Y la distinción entre los factores determinantes es un proceso necesario pero incompleto. Esta secuencia causal siempre es expost. El análisis debe llevar a este tipo de conclusiones: si en el momento anterior ocurrió $A$ entonces se presentó $B$.

Estas relaciones de causalidad no se pueden hacer sobre las relaciones futuras. Para Keynes es inaceptable afirmaciones del tipo: Si mañana
$C$ entonces sucederá $D . \mathrm{Y}$ estas interacciones causales no son pertinentes porque el futuro es incierto. Y para Hayek (1945) tales lógicas causales sobre dinámicas futuras son, por sí mismas, un germen de procesos autoritarios. La pretensión de predecir lógicas futuras puede ser la expresión de un afán de intervención, que niega la libertad humana. Para Hayek y los austriacos, el comportamiento endógeno -

4 Las críticas a la ilusión positiva las han hecho de manera radical, entre otros, los autores de la escuela austriaca. Ver, por ejemplo, Mises (1949); Hayek (1944, 1945, 1976, 1974), Horkheimer y Adorno (1944). 
cataláctico - de los miembros de la sociedad, va reconfigurando caminos impredecibles.

La desagregación de los fenómenos, y la definición de sus causalidades ex-post, es un ejercicio fundamental de todo proceso investigativo. En las disciplinas sociales, como en las naturales, la comprensión pasa por la formulación de secuencias causales. Y para entender estas dinámicas se puede recurrir a diferentes disciplinas. Marshall (1898) considera que la biología debería ser la ciencia auxiliar de la economía. En su opinión, la biología tiene la virtud de acercarse a la naturaleza del comportamiento humano.

Después de Samuelson (1983), las matemáticas se han convertido en el principal instrumento para la evaluación de la causalidad. Desde años antes del artículo de Samuelson, Keynes ya había advertido sobre el peligro del uso de las matemáticas, sin un adecuado enfoque apriorístico.

En gran medida, la reciente economía 'matemática' apenas es un brebaje, y es tan imprecisa como los supuestos iniciales sobre los que está basada, permitiendo que en una masa de símbolos inútiles y pretenciosos, el autor pierda de vista las complejidades e interdependencias del mundo real (Keynes, 1936, pos. 5236).

Las matemáticas, cuando no están acompañadas de prejuicios adecuados, terminan siendo un "brebaje". Y, finalmente, se convierten en una "masa de símbolos inútiles y pretenciosos". El abuso de las matemáticas ha impedido la reflexión sobre aspectos fundamentales de la disciplina. No hay duda de que en el desarrollo del análisis económico las matemáticas han jugado un papel importante. Mientras que Bentham (1965) necesitó páginas y páginas para explicar que, en el margen, la utilidad que depende de la riqueza es decreciente, gracias a las matemáticas actualmente se puede expresar la misma idea en dos líneas, diciendo que la primera derivada de la utilidad con respecto al ingreso es positiva, y que la segunda derivada
Las matemáticas, cuando no están acompañadas de prejuicios adecuados, terminan siendo un "brebaje". Y, finalmente, se convierten en una "masa de símbolos inútiles y pretenciosos".

es negativa. Las matemáticas son una herramienta que permite simplificar el lenguaje, pero cuando se aplican sin contexto apenas son un brebaje inútil, que desvía la atención, y no permite reflexionar con cuidado sobre las relaciones de causalidad de los fenómenos.

De manera general, las regresiones que se utilizan en economía indagan por causalidades lineales, tal y como se desprende de las relaciones de Granger (2003), o del teorema de las preferencias reveladas (Varían, 1990). En estas secuencias la lógica es acíclica: si $A$ explica $B, y B$ explica $C$, entonces $A$ explica $C y$, por tanto, no es cierto que $C$ explique $A$.

Más allá de la causalidad lineal, la hipótesis también podría optar por una causalidad circular (Hicks 1979). En tal caso la secuencia es cíclica: si $A$ explica $B, y B$ explica $C$, entonces A explica $C$, y también es posible que $C$ explique $A$. Este tipo de secuencia es frecuente en la realidad. Por ejemplo: el consumo depende del ingreso e, igualmente, el ingreso depende del consumo. Esta secuencia circular se puede plantear en un momento del tiempo, o en dos momentos diferentes. En los análisis monetarios el precio depende de la cantidad de dinero, pero también la cantidad de dinero depende del precio. La primera dirección de la causalidad se acerca a un enfoque como el de Friedman (1982). Y la segunda a una perspectiva keynesiana, ya que la cantidad de dinero responde a las estructuras de costos y a las necesidades de la economía.

Cualquiera que sea la forma de la causalidad, estas hipótesis son necesarias para avanzar en 
el conocimiento. Y son adecuadas si cumplen dos condiciones. La primera tiene que ver con que el saber apriorístico sea pertinente. Es decir, la hipótesis se debe enmarcar en los prejuicios iniciales, que deberían llevar a seleccionar un asunto que sea relevante. Y la segunda se refiere a la condición de parcialidad y relatividad del hallazgo. Nunca es el resultado final. Apenas es un componente de un conjunto más amplio, y este resultado parcial puede ser relativizado en la tercera fase, cuando se realizar la integración multicausal.

\section{La multicausalidad}

Y la tercera fase propuesta por Keynes consiste en reexaminar las causalidades obtenidas a través de la desagregación, con el fin de volver a la visión de conjunto, integrando los diversos componentes. Se trata, entonces, de superar los vínculos unicausales con el fin de realizar un análisis multicausal. Y este ejercicio final, de cierre, permite relativizar los hallazgos unidireccionales, y proponer alternativas interpretativas globales. La interacción entre factores es intrínsecamente compleja, y no se resuelve con el mejoramiento de las técnicas formales, sino con el desarrollo comprehensivo de la hipótesis. En su crítica a los autores clásicos, Keynes los acusa de realizar aproximaciones parciales, y de no haber logrado una lectura general. Por esta razón, su teoría es general.

$\mathrm{Al}$ tratar de entender las interrelaciones, las causalidades parciales que se encontraron en el segundo momento pierden capacidad explicativa porque quedan subsumidas en una interpretación más amplia. La causalidad $A$ explica $B$ se transforma en $A$ junto con otras variables, ayuda a explicar $B$. Y, por tanto, cualquier solución es inacabada, y los instrumentos de política económica no son definitivos, ni ofrecen soluciones milagrosas. Por tal razón, la política económica no puede actuar guiada por reglas, sino por decisiones discrecionales que se tienen que ir modificando de manera continua.

El simplismo de la regla lleva a afirmaciones como la independencia de los bancos centrales se traduce en menor inflación, el control de la masa monetaria reduce la inflación, el cumplimiento de la regla fiscal garantiza el crecimiento. Las reglas se han introducido en el análisis económico, sobre todo desde finales de los años 70s y están muy influenciadas por la visión de Friedman ${ }^{5}$. La lógica de la regla responde a relaciones de causalidad unidireccionales. La discreción, en cambio, obliga a la reflexión multicausal. Y las reglas suelen estar inscritas en modelos de estado estacionario, en los que se pierde la multicausalidad. Para que la dinámica sea estacionaria se requiere que el principio de ceteris paribus se mantenga durante todo el período de proyección.

La multicausalidad no permite construir modelos de estado estacionario, ya que las interacciones endógenas obligarían a utilizar modelos no lineales. En lugar de renunciar al equilibrio, y aceptar la multicausalidad, la llamada corriente principal ha preferido mantener los análisis en términos lineales, bajo el supuesto de unicausalidad.

Ningún modelo econométrico es capaz de explicar las complejidades inherentes a la visión multidimensional. De nuevo, es el sentido común el que logra ordenar en esta etapa final. La diferencia entre el primer momento y el último es el grado de información. El paso final, que busca la "interacción entre los factores", exige que el sentido común, ahora más informado, realice la jerarquización de hipótesis alternativas, y determine la que juzga más adecuada. La finalización del ejercicio, en esta última etapa, ya no permite mantener el supuesto de estado estacionario.

5 En el debate sobre reglas y discreción se destacan Barro (1986), Barro y Gordon (1983). Kydland y Prescott (1977), Modigliani (1964). 


\section{Verdad y conocimiento}

La verdad es parcial y es fruto del consenso informado. Una vez que se han realizado los tres pasos propuestos por Keynes, cabe la pregunta por la verdad del resultado. En el instante presente no es posible saber si los hallazgos son verdaderos. Este juicio quizás se pueda realizar ex-post.

Una vez que el estudiante, o el docente, han realizado los tres pasos anteriores, y terminan la investigación proponiendo una hipótesis final, viene el largo y agobiante proceso de crítica y autocrítica. Y el debate comienza desde el momento en el que los pares conocen los resultados y el método de la investigación. Si se logra un cierto consenso se puede afirmar que razonablemente, el resultado es verdadero (Sen, 1983; Sen, 1993).
Pero como la discusión entre visiones alternativas está marcada por los enfoques políticos, la fuerza argumentativa se puede debilitar, y el consenso de la llamada corriente principal se impone sin que medie una discusión relevante.

Como la discusión entre visiones alternativas está marcada por los enfoques políticos, la fuerza argumentativa se puede debilitar, y el consenso de la llamada corriente principal se impone sin que medie una discusión relevante.

\section{Conclusiones}

El tema de la enseñanza compete a la institución, al docente y al estudiante. Si se aceptan los tres pasos mencionados atrás, las tareas de cada uno de los agentes se pueden describir de la siguiente manera.

La institución - facultad - tiene la responsabilidad de incentivar el debate entre pares. Esta discusión tiene un doble propósito. Por un lado, crear los elementos contextuales que permitan examinar los prejuicios. La concreción de esta reflexión se expresa en el pensum, y en el peso que tengan las asignaturas que permitan avanzar hacia una visión amplia, y que estimulen una lectura cuidadosa de autores. Esta conclusión no es nueva, ha sido reiterada en las evaluaciones sobre la enseñanza de la economía ${ }^{6}$.

6 La enseñanza plural ha sido una conclusión reiterada de varios estudios. Ver, por ejemplo, Aebli (1987); Carr y Kemmis (1988); Bejarano (1978, 1997, $1999 a, b)$; Congote y Téllez (2017); Corredor y Castillo (2019); Cuenca (2010); Currie (1965
Y en Colombia las facultades de economía no solamente desprecian el debate al interior, sino que no están participando en la discusión nacional. Esta pasividad ha llevado a la prelación de visiones unilaterales, y de ejercicios empíricos simplistas, como el Marco Fiscal de Mediano Plazo 2020 (Ministerio de Hacienda 2020), que tienen enormes implicaciones en el terreno de la política pública.

El docente llega con sus prejuicios, y a partir de allí estructura sus cursos, y los proyectos de investigación. La docencia está fallando, sobre todo, en las etapas 1 y 3 del proceso cognitivo. Por un lado, numerosas preguntas son irrelevantes. Y, por el otro, la conclusión multicausal suele estar ausente, así que los cursos y las investigaciones terminan atrapadas en la reflexión sobre causalidades aisladas enfocadas en temas irrelevantes.

a, b); González (1996, 1999); Jaramillo (1951); Salazar (2019); Valencia y Muñetón (2004). 
El panorama es pesimista. El método unicausal está aniquilando la reflexión sistemática sobre problemas relevantes. En este proceso no solamente se empobrece el debate, sino que, además, se pierde el interés por la gran búsqueda de la que habla Nasar.

Los docentes suelen ser conservadores, y no van modificando su visión a medida que la teoría económica avanza. Hay un contraste marcado entre la diversidad de enfoques que se observan entre los premios Nobel, o en numerosos artículos y libros, y la incapacidad de los docentes de cambiar y de ir replanteando los criterios de la enseñanza y de la investigación.

El estudiante queda preso en la estrechez de la institución y los docentes. De vez en cuando hay desahogos y rebeliones, pero no se observan cambios sustantivos en la enseñanza (Español y De la Villa 2016). Los libros de texto de micro continúan pegados de los lineamientos ofrecidos por Samuelson (1983), hace más de 60 años. Y la macro todavía pretende entender el crecimiento a partir de desarrollos de la Cobb-Douglas. Sen (1998) critica la preocupación por el excesivo formalismo al que se ha llevado la función de producción, olvidando los temas que finalmente inciden en el crecimiento. El texto de Mankiw (2009) es un excelente ejemplo de esclerosis intelectual. $\mathrm{Al}$ estudiante se le cortan las alas desde el primer día cuando se le enseña que la curva de oferta tiene pendiente positiva, y la de demanda pendiente negativa. Desde finales del siglo XIX, Walras (1954) mostró que no era así. Su curva de oferta tiene un trayecto con pendiente positiva y otro con pendiente negativa.

La pésima enseñanza de la economía tiene los costos que se han hecho evidentes durante la pandemia. Las desigualdades se agudizan, y el principal problema que debería mover la gran búsqueda difícilmente permea a las facultades de economía. En los cursos de desarrollo nadie se pregunta ¿por qué el año pasado en el planeta Tierra había 265 millones de personas pasando hambre?

\section{Referencias}

Aebli, H. (1987). Factores de la enseñanza que favorecen el aprendizaje autónomo. Narcea.

Barro, R. (1986). Recent Developments in the Theory of Rules versus Discretion, Economic Journal, vol. 96, pp. 23-37.

Barro, R. y Gordon, D. (1983). Rules, Discretion, and Reputation in a Model of Monetary Policy, Journal of Monetary Economics, vol. 12, pp. 101-121.

Bejarano, J. (1978). Las Necesidades del Saber Histórico, en Bejarano, J. (2011). Antología Jesús Antonio Bejarano. Volumen 4. Estudios de Historia e Historiografía. Tomo 1. Historia Económica, Colección Obra Selecta, Universidad Nacional, pp. 17-22.

Bejarano, J. (1997). La Investigación Económica en Colombia, Cuadernos de Economía, vol. 16, no. 27, pp. 219-243.
Bejarano J. (1999a). Evaluación del Estado de la Disciplina Económica en Colombia: Un Enfoque Institucional, en BEJARANO Jesús., 2011. Antología Jesús Antonio Bejarano. Volumen 1. Estudios Económicos. Tomo 2. Teoría Económica e Instituciones, Colección Obra Selecta, Universidad Nacional, pp. 165-198.

Bejarano, J. (1999b). La Profesionalización en Economía, en BEJARANO Jesús., 2011. Antología Jesús Antonio Bejarano. Volumen 1. Estudios Económicos. Tomo 2. Teoría Económica e Instituciones, Colección Obra Selecta, Universidad Nacional, pp. 199-222.

Bentham, J., (1965). Defensa de la Usura", en STARK William., 1952, ed. Escritos Económicos. Jeremy Bentham, Fondo de Cultura Económica, pp. 194-226. 
Carr, W. y Kemmis, S. (1988). Teoría Crítica de la Enseñanza. La Investigación Acción en la Formación del Profesorado. Martínez Roca.

Cobb, Ch. y Douglas, P. (1928). A Theory of Production, American Economic Review, vol. 18, no. 2, mar., pp. 139-165.

Congote, B. y Tellez, M. (2017). "Cómo Mejorar la Enseñanza y el Aprendizaje de Teoría Económica Aplicando Algunas Herramientas Cuánticas", Tecnogestión, vol. 14 , no. 1, pp. 103-114.

Corredor, F. y Castillo, M. (2019). La Enseñanza de la Economía: Política, Ideología y Mercado, en GONZALEZ Jorge., PEREZ Mauricio., ed. Mercados y Bienestar. Ensayos en Memoria de Homero Cuevas, Universidad Externado.

Cuenca, M. (2010). La Enseñanza en Economía: Una Reflexión desde la Perspectiva del Contenido, Semestre Económico, vol. 13, no. 26, enero-jun., pp. 135-147.

Cuevas, H. (1986). Valor y Sistema de Precios, CID, Universidad Nacional.

Currie, L. (1965ª). La Enseñanza de la Economía (I): La Economía en un País en Desarrollo, Cuadernos de Economía, vol. 13, no. 18/19, 1993, pp. 330-343.

Currie,L. (1965 b). La Enseñanza dela Economía (II): Extractos de Informes sobre Algunas Facultades, Cuadernos de Economía, vol. 13, no. 18/19, 1993, pp. 345-354.

Duranton, G. y Puga, D. (2004). Micro-foundations of Urban Agglomeration Economies, en Herdenson Vernon., Thisse JacquesFrançois., ed. Handbook of Regional and Urban Economics, vol. 4, North Holland, pp. 2063-2117.

Edgeworth, F. (1879). The Hedonical Calculus, Mind, vol. 4, no. 15, jul., pp. 394-408.

Español, F. y De la Villa, L. (2016). Rebelión en las Aulas de Economía: Por Qué Poner Fin a la Monocultura Neoclásica, Oikonomics, no. 5, mayo.

Friedman, M. (1982). Teoría de los Precios, Alianza.
González, J. (1996). Desprofesionalización de la Enseñanza y Desarrollo del Saber Contable, Activos, no. 17, 2010, pp. 19-27.

González, J. (1999). La Fetichización del Currículo y la Absolutización del Libro de Texto, en BEJARANO Jesús., comp. Hacia Dónde Va la Ciencia Económica en Colombia. Siete Ensayos Exploratorios, Tercer Mundo, Colciencias, Universidad Externado, Bogotá, pp. 53-88.

Granger, C. (1969). Investigating Causal Relations by Econometric Models and Cross-Spectral Methods, Econometrica, vol. 37, no. 3, aug., pp. 424-438.

Granger, C. (2003). Time Series Analysis, Cointegration, and Applications, Nobel Lecture, University of California, San Diego.

Hayek, F. von. (1945). The Road to Serfdom with the Intellectuals and Socialism. Condensed Version, Reader's Digest, IEA.

Hayek, F. von., (1976). The Sensory Order. An Inquiry into the Foundations of Theoretical Psychology, University of Chicago Press, Chicago, 1976.

Hayek, F. von. (1978). "La Pretensión del Conocimiento", en Los Premios Nobel de Economía 1969-1977, Fondo de Cultura Económica.

Hayek, F. von. (2006). Camino de Servidumbre, Unión Editorial.

pp. 245-258.

Hicks, J. (1979). Causality in Economics, Oxford University Press.

Horkheimer, M. y Adorno, TH. (1944). Dialectic of Enlightenment. Philosophical.

Jaramillo J. (1994). En Torno a la Enseñanza de la Economía", Lecturas de Economía, no. 40, enero-jun., pp. 199-212.

Kahneman, D. (2002). Maps of Bounded Rationality: A Perspective on Intuitive Judgment and Choice, Nobel Lecture, Princeton University, Princeton, pp. 449-489.

Kahneman, D. (2011). Thinking, Fast and Slow, Penguin, New York, Kindle, Amazon. 
Kalmanovitz, S. (1999). El debate debe continuar: Bejarano y la Enseñanza de la Economía, Cuadernos de Economía, vol. 18, no. 31, pp. 173-198.

Keen, S. (2011). La Economía Desenmascarada, Capitán Swing, Madrid.

Keynes, J. M. (1936). The General Theory of Employment, Interest and Money, ClassicsUnbound.com, Kindle, Amazon.

Krugman, P. (1991). Geografía y Comercio, Antoni Bosch, Barcelona, 1992.

Kydland, F. y Prescott, E. (1977). Rules Rather than Discretion. The Inconsistency of Optimal Plans, Journal of Political Economy, vol. 85, no. 3, jun., pp. 473-492.

Mankiw, G. (2009). Macroeconomía, Antonio Bosch, Barcelona, 2014.

Marshall, A. (1898). Distribution and Exchange, Economic Journal, vol. 8, no. 29, mar., pp. 37-59.

Marshall, A. (1994). Principles of Economics. An Introductory Volume, eighth edition, Porcupine.

Ministerio de Hacienda y Crédito Público (2020). Marco Fiscal de Mediano Plazo 2020, Ministerio de Hacienda.

Mises, L. von (1960). La Acción Humana. Tratado de Economía, 2 vol., Fundación Ignacio Villalonga, Valencia.

Modigliani, F. (1964). Some Empirical Tests of Monetary Management and of Rules versus Discretion, Journal of Political Economy, vol. 72, no. 3, jun., pp. 211-245.

Nasar, S. (2011). Grand Pursuit. A Story of Economic Genius, Fourth Estate, Kindle, Amazon.

Piketty, Th. (2013). El Capital en el Siglo XXI, Fondo de Cultura Económica, México, 2014.

Piketty, Th. (2019). Capital e Ideología, Titivillus, ePub.
Salazar, B. (2019). El Pluralismo en la Enseñanza de la Economía, Ensayos de Economía, vol. 29, no. 55, jul.-dic., pp. 7-9.

Samuelson, P. (1937). A Note on Measurement of Utility. Review of Economic Studies, vol. 4, no. 2, feb., pp. 155-161.

Samuelson, P. (1983). Foundations of Economic Analysis, Harvard University Press.

Schumpeter, J. (1978). Historia del Análisis Económico, Ariel, España, 1994.ç VARIAN Hal., 1978. Análisis Microeconómico, Antoni Bosch.

Sen A. (1983). "Evaluator Relativity and Consequential Evaluation", Philosophy and Public Affairs, vol. 12, no. 2, spring, pp. 113-132.

Sen, A. (1998). Debates sobre Teoría del Capital. En Cuadernos de Economía, vol. 17, no. 29, pp. 27-38.

Sen, A. (1993). Positional Objectivity, Philosophy and Public Affairs, vol. 22, no. 2, spring, pp. 126-145.

Simon, H. (1978 a). Rational Decision Making in Business Organization, Nobel Lecture, American Economic Review, vol. 69, no. 4, pp. 493-513.

Simon, H. (1978b). "Rationality as Process and as a Product of Thought", American Economic Review, vol. 68, no. 2, may, pp. 1-16.

Thaler, R. (2017). From Cashews to Nudges: The Evolution of Behavioral Economics, Nobel Lecture, University of Chicago.

Varian, H. (1990). Análisis Microeconómico, Antoni Bosch.

Valencia, M. y Muñetón, G. (2004). Métodos de Enseñanza en Economía: Alternativas a la Tiza y el Tablero, Oikos, no. 18, nov., pp. 75-92.

Walras, L. (1954). Elements of Pure Economics, Allen and Unwin. 